\title{
Prediction of Skeletal Medial-Lateral for transfemoral ischial containment sockets
}

\author{
Michael P. Dillon, PhD; ${ }^{*}$ Richard G D. Fernandez, BSc(Hons); ${ }^{2}$ Bircan Erbas, PhD; ${ }^{3}$ Chris Briggs, PhD; ${ }^{4}$ \\ Matthew Quigley, MCPO(Hons) ${ }^{1}$ \\ Departments of ${ }^{1}$ Rehabilitation, Nutrition and Sport; ${ }^{2}$ Physiology, Anatomy and Microbiology; and ${ }^{3}$ Public Health, \\ College of Science, Health and Engineering, La Trobe University, Melbourne, Australia; ${ }^{4}$ Department of Anatomy and \\ Neuroscience, University of Melbourne, Melbourne, Australia; and Consultant Forensic Anthropologist, Victorian \\ Institute of Forensic Medicine, Southbank, Australia
}

\begin{abstract}
Accurate measurement of the pelvis is critical for well-fitting and comfortable ischial containment sockets. The "Skeletal Medial-Lateral (ML)" is intrusive and unreliable to measure in vivo. This study aimed to determine how accurately the Skeletal ML could be predicted and to identify which measurements were significant predictors. Computed tomography scans were randomly sampled from a cadaveric database $(n=$ 200). Inclusion criteria were age $>20 \mathrm{yr}$; lower-limb alignment that replicated the anatomical position; and no evidence of osteological trauma, implants, or bony growths. Multivariate linear regression models were developed to predict the Skeletal ML based on a suite of independent variables, including sex, body mass, and distance between pelvic landmarks. The regression model explained $76 \%$ of the variance in the Skeletal ML $(p<0.001)$. Variables that contributed significantly to the prediction of the Skeletal ML $(p<0.05)$ included body mass, sex, inter-greater trochanter distance, pelvic depth, and age. Significant predictors of the Skeletal ML dimension characterize variation in subcutaneous adipose tissue thickness and pelvic morphology. The Skeletal ML could be predicted with relatively small errors (standard error of estimate $=7 \mathrm{~mm}$ ) that could be easily and reliably adjusted during socket fitting. Further research is needed to test the predictive tool in a real-world setting.
\end{abstract}

Key words: amputation, anthropometry, computed tomography, ischial containment, pelvic morphology, prediction, prosthesis, Skeletal Medial-Lateral, socket, transfemoral.

\section{INTRODUCTION}

More than two-thirds of people living with transfemoral amputation report that problems with the fit of their prosthetic socket cause discomfort, impair mobility, and restrict participation in everyday activities [1-3].

Accurate measurement of the transfemoral residuum and pelvis is critical for a well-fitting and comfortable ischial containment socket. One of the most important measurements is the "Skeletal Medial-Lateral (ML)" because it defines the ML dimension of the socket brim that intimately encapsulates the pelvis and provides the necessary coronal plane stability to the remnant femur and pelvis that enables comfortable and effective gait [4-8]. Specifically, the Skeletal ML describes the coronal plane distance

\footnotetext{
Abbreviations: ASIS $=$ anterior superior iliac spine, $\mathrm{BMI}=$ body mass index, CT = computed tomography, GT = greater trochanter, ICC = intraclass correlation coefficient, $\mathrm{ML}=$ mediallateral, PSIS = posterior superior iliac spine, SEE = standard error of estimate, TEM = technical error of measurement.

*Address all correspondence to Michael P. Dillon, PhD; Department of Rehabilitation, Nutrition and Sport, College of Science, Health and Engineering, La Trobe University, Bundoora, 3086, Australia; +61-3-9479-5889.

Email: michael.dillon@latrobe.edu.au http://dx.doi.org/10.1682/JRRD.2015.03.0036
} 
between the medial aspect of the ischium and lateral shaft of the femur immediately inferior to the greater trochanter (GT) and includes the subcutaneous adipose tissue and skin [4-6]. Measurement of the Skeletal ML is difficult to obtain in vivo because of the need to accurately palpate anatomical features of the medial ischium and simultaneously locate a caliper in the intergluteal cleft. This is made more difficult as body mass and the thickness of subcutaneous adipose tissue increase.

Given an understanding of how the Skeletal ML is measured, it is not surprising that the technique is considered intrusive and that large variations in measurements are common [4]. We are not aware of any studies that have established the accuracy or reliability of the Skeletal ML measurement in vivo. Based on our clinical experience and teaching ischial containment to novice and experienced clinicians, we suggest that it is not uncommon for repeated measures to vary between as much as $1 / 2$ to $1 \mathrm{in}$. (12-25 mm). Similar variability (average range $12 \mathrm{~mm}$ ) was observed in a laboratory-based study using similar instrumentation, despite the relative simplicity of measuring a rigid foam model mounted in a bench vice [9]. A better technique is needed to improve the accuracy of the Skeletal ML measure without recourse to intrusive and unreliable measurement.

A pilot study on living subjects sought to determine the accuracy with which the Skeletal ML could be predicted using noninvasive measurements typically recorded by prosthetists during their everyday clinical practice, including sex, stature, anterior-posterior dimension (i.e., distance between the adductor longus tendon near its origin and the ischial tuberosity in sitting), and iliofemoral angle (i.e., soft tissue angle in the coronal plane between the lateral femoral shaft and gluteus medius in standing) [4]. While the regression model was able to explain 59 percent of the variance in the Skeletal ML, only sex contributed significantly to the prediction [4]. The standard error associated with the prediction was $9 \mathrm{~mm}$ [4]. Errors of this magnitude may be difficult to reliably adjust by packing (i.e., placing a foam piece inside the socket to fill a void or increase tissue loading) or heating the socket to alter its dimensions.

The error associated with predicting the Skeletal ML could be reduced by developing a predictive model using more precise input data from computed tomography (CT) and identifying additional independent variables that might explain a proportion of the variance not already described by sex. Where possible, independent variables should be bone-to-bone measurements given their improved accuracy and reliably compared with soft-tissue measures [10].

We sought to identify additional independent variables from the related gynecological and anthropological literature because anthropometric measurements of the pelvis have been widely used to identify women at risk for complicated delivery or to identify human remains. Any number of measures have been used to characterize differences in pelvic morphology, including pubic arch angle, diameter of the pelvic inlet, pelvic depth (i.e., anterior-posterior distance between the pubic tubercles and posterior superior iliac spines [PSISs]), and distance between the anterior superior iliac spine (ASIS) (Figure 1) [11-13]. Whether these measurements are strongly correlated with the Skeletal ML or account for the unique variance not already described by sex is unknown. A more pragmatic approach would be to look at measurements that clinicians could easily and reliably record during clinical practice and simply test these. To this end, the following independent variables were tested: sex, age, stature, body mass, and pelvic height and depth, as well as the distance between the ASIS, PSIS, iliac crests, and GT (Figure 1).

The aim of this study was to estimate the accuracy with which the Skeletal ML could be predicted and identify which independent variables contributed significantly to the prediction.

\section{METHODS}

\section{Selection of Computed Tomography Scans}

Whole body CT scans were obtained from a database of deceased persons at the Victorian Institute of Forensic Medicine, Melbourne, Australia. Ethics approval was provided by the Victorian Institute of Forensic Medicine, Research Advisory and Ethics Committee, as well as the La Trobe University Human Ethics Committee. The dataset was thought to be representative of the general population in that all persons undergoing some form of investigation following death (e.g., routine autopsy) were included in the database.

CT scans were randomly sampled from the database and subsequently vetted. Based on previous work [4], 200 cases were needed to detect a 0.13 change of slope for various predictors in a regression model with 80 percent power. Cases were defined using the following exclusion 


Description
Inter ASIS distance:
Distance between the most anterior points of
the left and right ASIS as measured in the
coronal plane.

Inter PSIS distance:
Distance between the most posterior points
of the left and right PSIS, as measured in the
coronal plane.

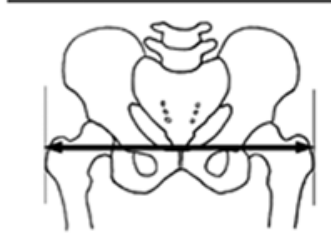

Inter greater trochanter distance: Distance between the lateral most projection of the left and right Greater Trochanters, as measured in the coronal plane

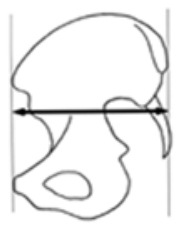

\section{Pelvic depth:}

Distance between two parallel reference lines defined by: 1 . the most anterior projections of the right and left pubic tubercles and 2 . the most posterior projection of the Posterior Superior Iliac Spines. Distance recorded in the sagittal plane.

Figure 1.

Schematic representation of pelvic measurements used as independent variables in predictive model. ASIS = anterior superior iliac spine, PSIS = posterior superior iliac spine.

criteria: younger than $20 \mathrm{yr}$ of age given incomplete pelvic growth [14-15], body mass and stature outside the Australian population norm [16], evidence of osteological trauma, orthopedic implants or bony growths (e.g., osteophyte formation), and lower-limb alignment atypical of the standing anatomical position (e.g., hip abduction).

\section{Apparatus}

CT scans were measured using proprietary software, Vitrea version 3.1 (Vital Images Inc; Minnetonka, Minnesota), where each frame/graduation corresponded to a $2 \mathrm{~mm}$ transverse slice thickness. The software included a rendered three-dimensional image showing the CT section being simultaneously displayed in each anatomical plane of reference.

\section{Procedure}

Details of sex, age, stature, and body mass were extracted from the case demographics reported with each CT scan. Anatomical planes of reference were subsequently established. The transverse plane was defined by the most inferior aspect of the left and right ischial tuberosities. The sagittal plane was defined by a plane passing through the most anterior part of the right and left ASIS and the pubic tubercles [17]. The coronal plane was perpendicular to the sagittal plane. This made it possible to pan through sequential slices of the CT scans in anatomical planes of reference and record measurements with respect to these planes (e.g., the inter-ASIS distance was measured in the coronal plane).

A variety of techniques was necessary to record measurements from the CT scans using the Vitrea ruler tool. In many cases, measurements were recorded between anatomical landmarks on the same slice of the CT scan (e.g., distance between the most anterior part of the right and left ASIS, Figure 1). Some measurements first required the establishment of reference lines to define bilateral landmarks. For example, pelvic depth was measured in the sagittal plane between two reference lines - that is, a reference line between the most anterior projection of the right and left pubic tubercles and a reference line between the most posterior projections of the right and left PSIS (Figure 1). In terms of the Skeletal ML, the points of measurement were not specifically defined by anatomical landmarks, and as such it was necessary to define these. A point of measurement on the medial ischium was defined by the intersection of two reference lines. The first reference line was midway between the most inferior part of the ischial tuberosity and most inferior part of the obturator foramen (Figure 2). The second reference line was at the midpoint between the most posterior part of the ischium and the most posterior part of the obturator foramen (Figure 2). These reference lines not only defined the point of measurement on the medial ischium but also the coronal and transverse plane slice at which the Skeletal ML would be measured. 
The Skeletal ML was recorded in three parts to assess concerns that the supine positioning of cadavers on the CT scanner would deform the posterior-lateral subcutaneous adipose tissue and compromise the Skeletal ML measurement. The three component parts of the Skeletal ML were the coronal plane distances from the (1) medial ischium to the lateral shaft of the femur; (2) lateral shaft of femur to the lateral margin of the iliotibial tract; and (3) iliotibial tract to the epidermis, encompassing the subcutaneous adipose tissue and skin (Figure 3).

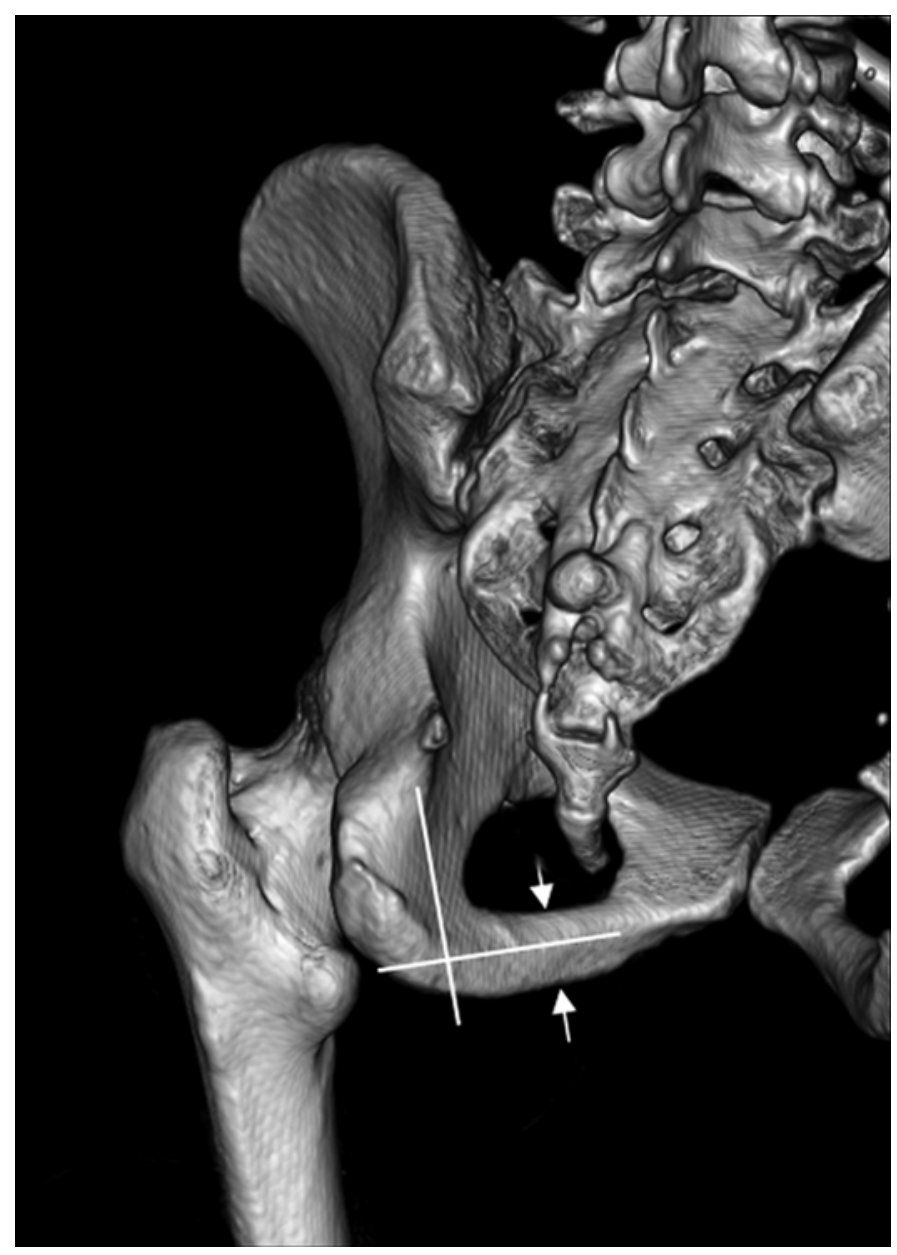

Figure 2.

Posterior-medial view of left ischium showing intersection of reference lines defining point at which Skeletal Medial-Lateral was measured. Horizontal reference line was midway between most inferior portion of ischial tuberosity and most inferior portion of obturator foramen (illustrated). Vertical reference line is midway between most posterior part of ischium and most posterior part of obturator foramen.
To determine whether the deformation of subcutaneous adipose tissue and skin would affect measurement of the Skeletal ML, we randomly sampled 20 cases, thereby ensuring that characteristics of sex, body mass index (BMI), stature, and age were representative. Based on this sample, we observed considerable deformation of the subcutaneous adipose tissue in many cases (Figure 4). Measures of subcutaneous adipose tissue and skin lateral to the iliotibial tract were not well correlated with BMI $\left(r^{2}=0.374, p=0.10\right)$, as would be expected based on related literature [18-19], and when used as part of the Skeletal ML measure did not result in typical sex differentiation between males $(0.149 \pm 0.013 \mathrm{~m})$ and females $(0.142 \pm 0.026 \mathrm{~m})$ [4]. The Skeletal ML was also considerably larger in females and disproportionately variable compared with that previously reported [4].

Given our concern that deformation of the posteriorlateral subcutaneous adipose tissue and skin resulted in erroneous measurements of the Skeletal ML, we tested

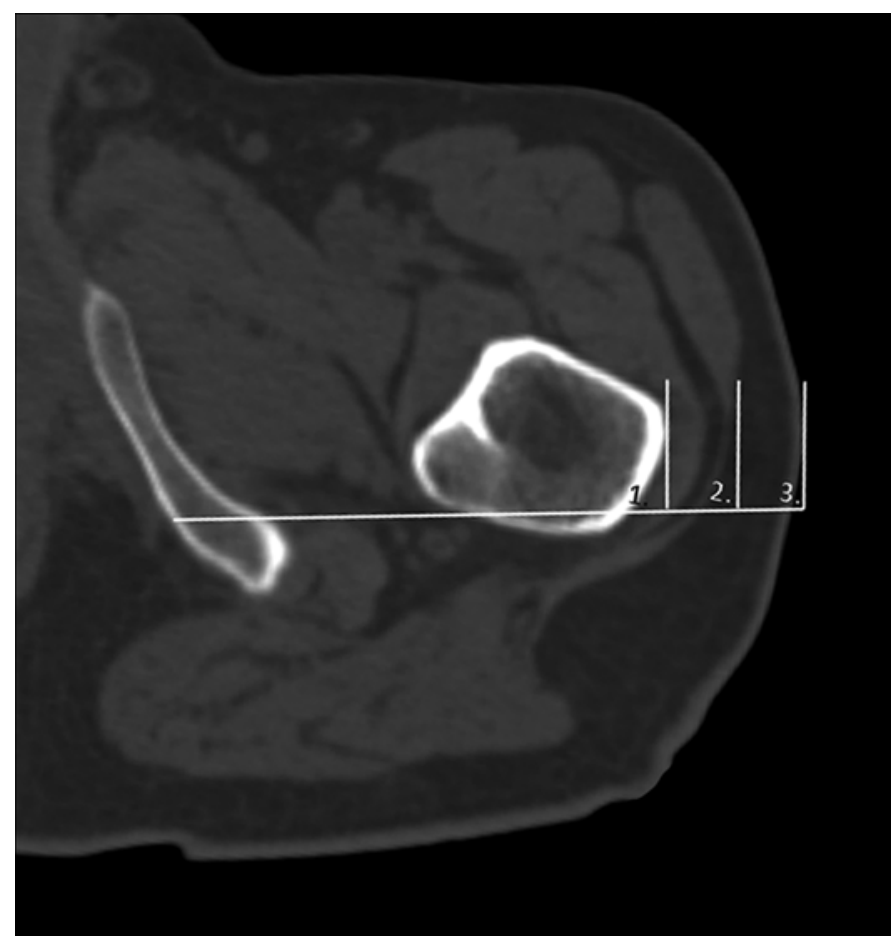

Figure 3.

Transverse plane computed tomography scan showing component parts of Skeletal Medial-Lateral measurement: (1) medial ischium to lateral shaft of femur, (2) lateral shaft of femur to lateral margin of iliotibial tract, (3) subcutaneous adipose and skin lateral to iliotibial tract. 


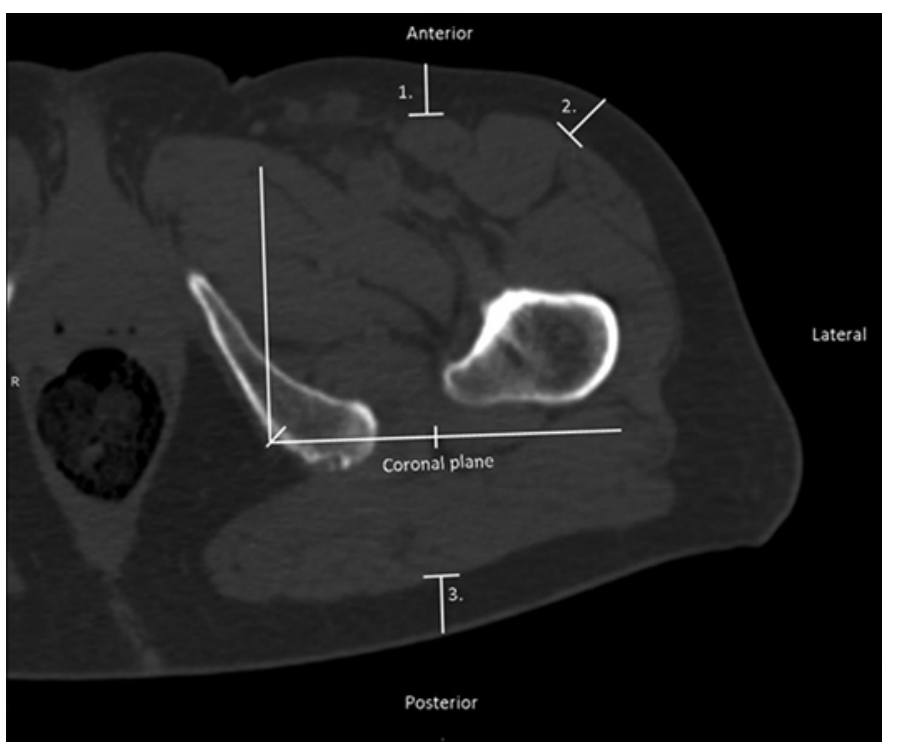

Figure 4.

Alternative locations trailed for measuring subcutaneous adipose and skin thickness (1) anteriorly, along sagittal plane reference line bisecting midpoint between medial ischium and lateral shaft of femur; (2) anterolaterally, along reference line $45^{\circ}$ to midsagittal and coronal plane; (3) posteriorly, along sagittal plane reference line bisecting midpoint between medial ischium and lateral shaft of femur.

additional locations for measuring the subcutaneous adipose tissue and skin thickness: (1) anteriorly, along the sagittal plane reference line bisecting the midpoint between the medial ischium and lateral shaft of femur; (2) anterolaterally, along a reference line $45^{\circ}$ to the midsagittal and coronal planes; and (3) posteriorly, along a sagittal plane reference line bisecting the midpoint between the medial ischium and lateral shaft of the femur (Figure 4). Along these reference lines, subcutaneous adipose tissue and skin were measured from the epidermis to the most superficial border of the musculature (Figure 4). Of these measures, only the anterior subcutaneous adipose tissue and skin thickness were correlated with BMI $\left(r^{2}=0.604, p=0.005\right)$ and resulted in typical sex differentiation and variability in males $(0.143 \pm 0.013 \mathrm{~m})$ and females $(0.118 \pm 0.013 \mathrm{~m})$, in keeping with previous literature $[4,18]$. As such, we used the anterior measure of subcutaneous adipose tissue and skin as an alternative to the lateral measure that was compromised by supine positioning of the cadavers.

The author (M.Q.) was trained to use Vitrea, and reliability of the measurements were subsequently assessed using a random sample of CT scans prior to the com- mencement of the study. CT scans were measured twice, 2 wk apart, without knowledge of the original measurements. The test-retest reliability was determined by intraclass correlation coefficients (ICCs) using a one-way random effects model and absolute agreement definition. All bone-to-bone (ICC $>0.9, p<0.001$ ) and soft tissue (ICC $>0.8, p<0.01$ ) measurements were reliable.

\section{Data Reduction and Analysis}

A hierarchical multivariate linear regression model was developed, in accordance with the techniques described by Pallant [20], to determine the accuracy of the Skeletal ML prediction. Separate regression models were developed to determine whether there were differences in the standard error and predictors of the Skeletal ML based on sex.

Preliminary analyses were conducted to ensure that the assumptions of normality and linearity were not violated so as to establish the validity of the linear regression [20]. The preliminary analyses, sample descriptive statistics, and linear regressions were computed using SPSS version 21 (IBM Corporation; Armonk, New York). The results were presented as standardized coefficients (beta) to allowed direct comparison of which independent variables had the greatest effect on the Skeletal ML.

\section{RESULTS}

Of the 200 cases measured, 111 were male (55\%) and 89 were female (45\%). Demographic data describe a wide cross-section of the population as evidenced by the range in age, stature, body mass, and BMI (Table 1). The average Skeletal ML was larger in males $(0.138 \pm 0.011 \mathrm{~m}$, range: $0.106-0.167 \mathrm{~m})$ than in females $(0.120 \pm 0.011 \mathrm{~m}$, range: $0.091-0.149 \mathrm{~m}$ ), and variability was comparable between sexes.

The regression model explained 76 percent of the variance in the Skeletal ML $\left(r^{2}=0.762\right)$, which was statistically significant $\left(F_{(10,189)}=60.524, p<0.001\right)$. A number of independent variables made a unique and statistically significant contribution to the regression model: body mass, sex, inter-GT distance, pelvic depth, and age (Table 2). The standard error associated with the prediction of the Skeletal ML was $0.07 \mathrm{~m}$ (standard error of estimate $[\mathrm{SEE}]=0.07$ ).

Separate regression models were developed to determine whether there were differences in the predictors 
JRRD, Volume 53, Number 2, 2016

Table 1.

Demographic characteristics of sample of computed tomography scans by sex.

\begin{tabular}{|c|c|c|c|c|c|c|c|c|c|c|}
\hline \multirow{2}{*}{ Demographic } & \multicolumn{5}{|c|}{ Females $(n=89)$} & \multicolumn{5}{|c|}{ Males $(n=111)$} \\
\hline & Mean & Median & SD & Min & Max & Mean & Median & SD & Min & Max \\
\hline$\overline{\text { Age (yr) }}$ & 58 & 57 & 19 & 24 & 94 & 59 & 58 & 17 & 22 & 93 \\
\hline Mass (kg) & 65.6 & 66.0 & 10.8 & 44.0 & 92.0 & 77.4 & 75.0 & 12.5 & 55.0 & 106.0 \\
\hline BMI $\left(\mathrm{kg} / \mathrm{m}^{2}\right)$ & 25.5 & 24.7 & 4.2 & 18.2 & 38.3 & 26.4 & 25.9 & 4.2 & 19.0 & 38.0 \\
\hline
\end{tabular}

Table 2.

Estimated coefficients from multivariate linear regression of Skeletal Medial-Lateral.

\begin{tabular}{lccc}
\hline \multicolumn{1}{c}{ Measure } & $\begin{array}{c}\text { Standardized } \\
\text { Coefficient } \\
\text { (Beta) }\end{array}$ & p-Value & $\begin{array}{c}\text { Semipartial } \\
\text { Correlation } \\
\text { Coefficient }\end{array}$ \\
\hline Mass & 0.549 & $<0.001$ & 0.460 \\
Sex & 0.337 & $<0.001$ & 0.199 \\
Inter-GT Distance & 0.303 & $<0.001$ & 0.213 \\
Pelvic Depth & -0.182 & $<0.001$ & -0.126 \\
Age & 0.110 & 0.02 & 0.086 \\
Interiliac Crest & 0.066 & 0.31 & 0.036 \\
Pelvic Height & -0.062 & 0.33 & -0.035 \\
Inter-ASIS Distance & 0.040 & 0.49 & 0.024 \\
Inter-PSIS Distance & -0.009 & 0.85 & -0.007 \\
Stature & 0.005 & 0.94 & 0.003 \\
\hline ASIS = anterior superior iliac spine, GT = greater trochanter, PSIS = posterior \\
superior iliac spine.
\end{tabular}

between sexes. In the female cohort, the regression model explained 60 percent of the variance in the Skeletal ML $\left(r^{2}=0.604\right)$, which was statistically significant $\left(F_{(9,79)}=13.387, p<0.001\right)$. A number of variables contributed significantly to the prediction in the female cohort, including mass (beta $=0.620, p<0.001$ ), interGT distance (beta $=0.468, p<0.001$ ), and pelvic depth (beta $=-0.210, p=0.045$ ). In the male cohort, the regression model explained a similar proportion of the variance in the Skeletal ML $\left(r^{2}=0.635\right)$, which was also statistically significant $\left(F_{(9,101)}=19.544, p<0.001\right)$. The same predictors were also significant contributors in the male sample: mass (beta $=0.641, p<0.001$ ), inter-GT distance (beta $=0.311, p<0.001$ ) and pelvic depth (beta $=-0.242$, $p=0.007$ ), with the addition of age (beta $=0.189, p=$ $0.02)$. These regression models were able to predict the Skeletal ML with a standard error of $0.07 \mathrm{~m}$ in both the females $(\mathrm{SEE}=0.07)$ and males $(\mathrm{SEE}=0.07)$.

\section{DISCUSSION}

This investigation sought to estimate how accurately the Skeletal ML could be predicted and to identify which variables contributed significantly to the prediction.

A number of independent variables - body mass, sex, pelvic depth, inter-GT distance, and age-made a unique and significant contribution to the regression model. The standard error associated with the prediction was $7 \mathrm{~mm}$, with no differences between sexes.

Of the independent variables that explained significant variation in the Skeletal ML, we suggest that sex, pelvic depth, and inter-GT distance characterize different shapes of the pelvic outlet. For example, the inter-GT distance may characterize the wide ML dimension of the platypelloid pelvis, and a large pelvic depth measure may typify the large anterior-posterior dimension of the anthropoid pelvis (Figure 5).

Body mass had the largest influence on the Skeletal ML, explaining 21 percent of the unique variance as determined by the square of part correlation coefficients reported in Table 2. It is logical that increased body mass also increased the Skeletal ML given that body mass was positively correlated with the subcutaneous adipose tissue and skin thickness component of the Skeletal ML in both males $\left(r^{2}=0.64, p<0.001\right)$ and females $\left(r^{2}=0.65\right.$, $p<0.001$ ) and that subcutaneous adipose tissue and skin thickness was a large proportion of the Skeletal ML (mean: 11\%, range: 4\%-35\%).

We were surprised that age explained a unique and significant part of the variation in Skeletal ML in males, particularly given that our sample only included cases that were skeletally mature. The observed relationship between increased age and pelvic width has been reported in just one other investigation, in which increased width between the GT and iliac wings was correlated with increasing age in both sexes [21]. Investigators have rejected the hypothesis 


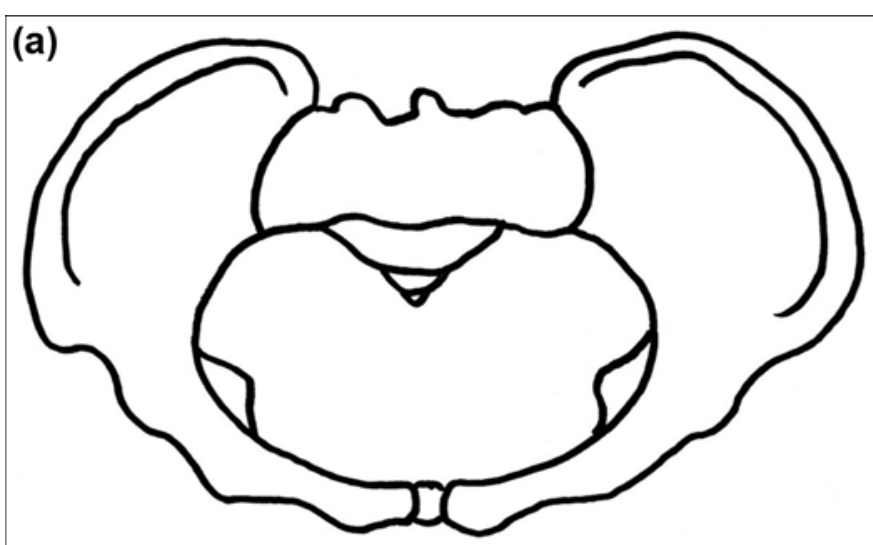

(b)

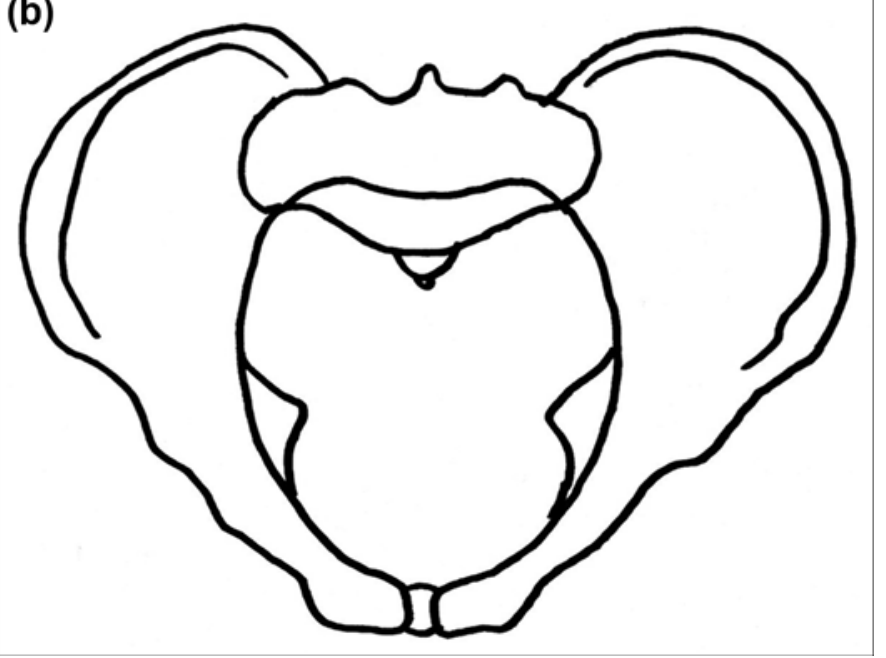

Figure 5.

Schematic of (a) platypelloid and (b) anthropoid pelvis types. Platypelloid pelvis shows wider mediolateral dimension, while anthropoid pelvis shows greater pelvic depth, as seen in anteriorposterior dimension.

that increased width between pelvic landmarks was due to periosteal apposition secondary to age-related decline in muscle mass and strength or endocortical resorption in postmenopausal women [21-22]. We tested whether inadvertent selection of older individuals with larger body mass had biased the sample, but the relationship between age and body mass in both the males $\left(r^{2}=0.014, p=0.15\right)$ and females $\left(r^{2}=0.115, p=0.28\right)$ was weak and not significant. As such, we are unable to explain the influence of age on the Skeletal ML.

Comparison with other studies was limited because only one other investigation has predicted the Skeletal ML
[4]. The average Skeletal ML measurements reported in this study (males: $0.138 \pm 0.011 \mathrm{~m}$, females: $0.120 \pm$ $0.011 \mathrm{~m}$ ) were comparable with previous in vivo measurement [4] (males: $0.140 \pm 0.090 \mathrm{~m}$, females: $0.119 \pm$ $0.080 \mathrm{~m}$ ), which engenders confidence that measurements recorded using CT scans provide similar outcomes. The regression model developed in this study explained an additional 17 percent of the variance in the Skeletal ML $\left(r^{2}=0.76\right)$ compared with previous work $\left(r^{2}=0.59\right)$ [4]. This could be attributed to more accurate input data from CT scans (compared with in vivo measurement), inclusion of additional independent variables that explain the variance in the Skeletal ML not previously described by sex, and a more appropriately powered study. In combination, these methodological differences explain the reduction of the standard error in this study $(\mathrm{SEE}=0.07 \mathrm{~m}$ ) compared with previous work (SEE $=0.09 \mathrm{~m})$ [4]. Difference in the standard error between these studies was modest despite the fact that we were able to explain a very high proportion of the variance in the Skeletal ML. While there will be other independent variables that explain part of the variance in the Skeletal ML, it is doubtful whether incremental increases in the coefficient of determination will dramatically reduce the standard error and further improve the accuracy of the prediction.

It could be argued that the real value of being able to predict the Skeletal ML lies not in its millimeter precision, but as an alternative to in vivo measurement where repeated palpation of the medial ischium and caliper placement in the intergluteal cleft near the anus is uncomfortable and intrusive.

Further work is necessary to demonstrate if the Skeletal ML prediction is sufficiently accurate for clinical use and that errors can be easily and reliably adjusted during check socket fitting.

\section{Limitations}

At the outset of the investigation, we expected that CT scans would be far more accurate and reliable than in vivo measurement. We had not anticipated the extent to which CT scans in supine would result in deformation of posterior-lateral subcutaneous adipose tissue and compromise measurement of the Skeletal ML. To address this, we measured the thickness of the subcutaneous adipose tissue and skin on the anterior thigh as a component of our Skeletal ML measurement as an alternative. We hope to have been clear about the rationale and careful to demonstrate the reasonableness of this alternative approach. As we 
have observed in this study, obtaining measurements from CT scans and in living subjects have inherent limitations and we can only expect similarity in measurements between different methods given there is no gold standard. The fact that the Skeletal ML measures reported in this study were comparable to those reported from the only in vivo study [4] helps engender confidence in the reasonableness of the approach.

Cadaver placement on the bed of the CT scanner was highly variable because of the presentation of deceased persons to the Victorian Institute of Forensic Medicine. While we were careful to address this through vetting of cases and our method of establishing anatomical planes of references for each scan, we did not control for variation in the femoral adduction/abduction angle or the degree of hip internal/external rotation beyond visually observing that the lower limbs were in a normal anatomical alignment. Theoretically, large changes in the femoral adduction/abduction angles or hip internal/external rotation would be needed to influence the Skeletal ML, and some variation is to be expected given that persons with limb loss, like the general population, present with a variety of lower-limb alignments. We acknowledge this as a source of uncontrolled variation in our measurement of the dependent variable, and future studies may determine that controlling for this variation is important.

While our sample included a wide cross-section of the population, there is no evidence to suggest the results would be applicable to children or adolescents given differences in pelvic morphology compared with the skeletally mature adults included in this sample.

\section{Clinical Implications}

Further work is necessary to put this predictive tool in the hands of clinicians and demonstrate its usefulness in a real-world setting. To this end, we are developing an application (iPhone app) that will allow clinicians to enter patient details (body mass, sex, age, width between the GT, and pelvic depth) to estimate the Skeletal ML. Clinicians can then use the estimated Skeletal ML as an alternative to in vivo measurement in their casting, cast evaluation, cast rectification, and socket fitting.

It will be important to evaluate the reliability with which clinicians can measure the input parameters and the effect of these variations on the accuracy of the Skeletal ML prediction. The first step in this process would be to develop a protocol to ensure that prosthetists adopt reliable practices for the measurement of the anthropo- metric input data. When such protocols have been utilized, measurement of body mass and inter-GT distances have been shown to be reliable and have a small technical error of measurement (TEM) [23-24]. As an illustrative example, the intrarater TEM associated with the inter-GT distance is $0.5 \mathrm{~cm}$ [23]. Given that we were unable to find evidence of the TEM associated with pelvic depth and that measurement error varies based on the population and instrumentation, a specific protocol would need to be developed and tested as part of any further work. It will also be important to determine whether ischial containment sockets made using the predictive technique result in fewer or less significant errors in the Skeletal ML that require adjustment or new sockets.

\section{CONCLUSIONS}

This investigation shows that the Skeletal ML can be predicted with a small standard error using body mass, sex, inter-GT distance, pelvic depth, and age. These predictors characterize differences in subcutaneous adipose tissue thickness and pelvic morphology that underlie variation in the Skeletal ML. The relatively small errors associated with the prediction could be easily and reliably adjusted during fitting of the socket. Further research is needed to demonstrate the usefulness of the predictive tool in a real-world setting and evaluate whether ischial containment sockets made using the predictive tool result in fewer or less significant errors in the Skeletal ML compared with in vivo measurement.

\section{ACKNOWLEDGMENTS}

\section{Author Contributions:}

Study concept and design: M. P. Dillon. Acquisition of data: M. Quigley, R. G. D. Fernandez, M. P. Dillon. Analysis and interpretation of data: M. P. Dillon, B. Erbas.

Drafting of manuscript: M. P. Dillon, M. Quigley. Critical revision of manuscript for important intellectual content: R. G. D. Fernandez, B. Erbas, C Briggs.

Obtained funding: M. P. Dillon, R. G. D. Fernandez, B. Erbas, C. Briggs. Financial Disclosures: The authors have declared that no competing interests exist.

Funding/Support: This research was funded by the research focus area in sport, exercise and rehabilitation, La Trobe University (Principal Investigator: Michael Dillon).

Additional Contributions: The authors wish to thank the Victorian Institute of Forensic Medicine for their collaboration. 
Institutional Review: Ethics approval was provided by the Victorian Institute of Forensic Medicine, Research Advisory and Ethics Committee as well as the La Trobe University Human Ethics Committee.

\section{REFERENCES}

1. Meulenbelt HE, Geertzen JH, Jonkman MF, Dijkstra PU. Determinants of skin problems of the stump in lower-limb amputees. Arch Phys Med Rehabil. 2009;90(1):74-81. [PMID:19154832] http://dx.doi.org/10.1016/j.apmr.2008.07.015

2. Dudek NL, Marks MB, Marshall SC, Chardon JP. Dermatologic conditions associated with use of a lower-extremity prosthesis. Arch Phys Med Rehabil. 2005;86(4):659-63. [PMID:15827914] http://dx.doi.org/10.1016/j.apmr.2004.09.003

3. Dillingham TR, Pezzin LE, MacKenzie EJ. Limb amputation and limb deficiency: Epidemiology and recent trends in the United States. South Med J. 2002;95(8):875-83. [PMID:12190225]

4. Dillon MP, Laux S, Fernandez RG. Prediction of the skeletal medio-lateral dimension using non-invasive anthropometric measurements for the provision of ischial containment sockets: A pilot study. Prosthet Orthot Int. 2014;38(2):133-39. [PMID:23798041] http://dx.doi.org/10.1177/0309364613490442

5. Dillon MP. Ischial containment socket for transfemoral amputees. A manual for assessment, casting, modification and fitting. Bundoora (Australia): National Centre for Prosthetics and Orthotics, La Trobe University; 2006.

6. Hoyt C, Littig D, Lundt J, Statts TB. The UCLA CATCAM above-knee prosthesis. 3rd ed. Los Angeles (CA): UCLA Prosthetics Education and Research Program; 1987.

7. Sabolich J. Contoured adducted trochanteric controlled alignment method (CAT-CAM). Clin Prosth Orthot. 1985; 9(4):15-26.

8. Pritham $\mathrm{CH}$. Biomechanics and shape of the above-knee socket considered in light of the ischial containment concept. Prosthet Orthot Int. 1990;14(1):9-21. [PMID:2192356]

9. Geil MD. Consistency and accuracy of measurement of lower-limb amputee anthropometrics. J Rehabil Res Dev. 2005;42(2):131-40. [PMID:15944877] http://dx.doi.org/10.1682/JRRD.2004.05.0054

10. Cameron N. Measuring growth. In: Hauspie RC, Cameron $\mathrm{N}$ Molinari L, editors. Methods in human growth research. Cambridge (United Kingdom): Cambridge University Press; 2004.

11. Tague RG. Variation in pelvic size between males and females. Am J Phys Anthropol. 1989;80(1):59-71.
12. Washburn SL. Sex differences in the pubic bone. Am J Phys Anthropol. 1948;6(2):199-208.

13. Ridgeway B, Arias BE, Barber MD. The relationship between anthropometric measurements and the bony pelvis in African American and European American women. Int Urogynecol J. 2011;22(8):1019-24. [PMID:21468737] http://dx.doi.org/10.1007/s00192-011-1416-1

14. Rissech C, García M, Malgosa A. Sex and age diagnosis by ischium morphometric analysis. Forensic Sci Int. 2003; 135(3):188-96. [PMID:12927396] http://dx.doi.org/10.1016/S0379-0738(03)00215-9

15. Lee MC, Eberson CP. Growth and development of the child's hip. Orthop Clin North Am. 2006;37(2):119-32, v. [PMID:16638443] http://dx.doi.org/10.1016/j.ocl.2005.12.001

16. Australian Bureau of Statistics. How Australians measure up. Canberra (Australia): Australian Bureau of Statistics; 1995.

17. McKibbin B. Anatomical factors in the stability of the hip joint in the newborn. J Bone Joint Surg Br. 1970;52(1): 148-59. [PMID:5436200]

18. Akkus O, Oguz A, Uzunlulu M, Kizilgul M. Evaluation of skin and subcutaneous adipose tissue thickness for optimal insulin injection. J Diabetes Metab. 2012;3(8):216-21.

http://dx.doi.org/10.4172/2155-6156.1000216

19. Gibney MA, Arce CH, Byron KJ, Hirsch LJ. Skin and subcutaneous adipose layer thickness in adults with diabetes at sites used for insulin injections: Implications for needle length recommendations. Curr Med Res Opin. 2010;26(6): 1519-30. [PMID:20429833] http://dx.doi.org/10.1185/03007995.2010.481203

20. Pallant J. SPSS survival manual. Crows Nest (Australia): Allen \& Unwin; 2005.

21. Berger AA, May R, Renner JB, Viradia N, Dahners LE. Surprising evidence of pelvic growth (widening) after skeletal maturity. J Orthop Res. 2011;29(11):1719-23.

[PMID:21608025]

http://dx.doi.org/10.1002/jor.21469

22. Szulc P, Seeman E, Duboeuf F, Sornay-Rendu E, Delmas PD. Bone fragility: Failure of periosteal apposition to compensate for increased endocortical resorption in postmenopausal women. J Bone Miner Res. 2006;21(12):1856-63. [PMID:17002580] http://dx.doi.org/10.1359/jbmr.060904

23. Frisancho AR. Anthropometric standards for the assessment of growth and nutritional status. Ann Arbor (MI): University of Michigan Press; 1990.

24. Ulijaszek SJ, Kerr DA. Anthropometric measurement error and the assessment of nutritional status. Br J Nutr. 1999; 82(3):165-77. [PMID:10655963] http://dx.doi.org/10.1017/S0007114599001348 
JRRD, Volume 53, Number 2, 2016

Submitted for publication March 3, 2015. Accepted in revised form July 6, 2015.

This article and any supplementary material should be cited as follows:

Dillon MP, Fernandez RG, Erbas B, Briggs C, Quigley M. Prediction of Skeletal Medial-Lateral for transfemoral ischial containment sockets. J Rehabil Res Dev. 2016; 53(2):253-62.

http://dx.doi.org/10.1682/JRRD.2015.03.0036
ORCID: Michael P. Dillon, PhD: 0000-0003-3614-7787; Richard G. D. Fernandez, BSc(Hons): 0000-0002-20878633; Matthew Quigley, MCPO(Hons): 0000-0002-58718993

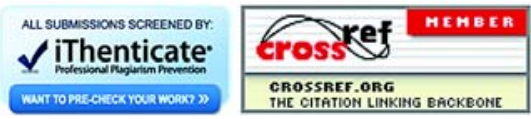

